\title{
A Parameterized Geometric Magnetic Field Calibration Method for Vehicles with Moving Masses with Applications to Underwater Gliders
}

\author{
Brian Claus \\ Applied Ocean \\ Physics and Engineering \\ Woods Hole Oceanographic Institute \\ Woods Hole, MA \\ bclaus@whoi.edu
}

\author{
Ralf Bachmayer \\ Department of Ocean and \\ Naval Architecture Engineering \\ Memorial University \\ St. John's, NL \\ bachmayer@mun . ca
}

\begin{abstract}
The accuracy of magnetic measurements performed by autonomous vehicles is often limited by the presence of moving ferrous masses. This work presents a parameterized ellipsoid field calibration method for magnetic measurements in the sensor frame. In this manner the ellipsoidal calibration coefficients are dependent on the locations of the moving masses. The parameterized calibration method is evaluated through field trials with an autonomous underwater glider equipped with a low power precision fluxgate sensor. A first set of field trials were performed in the East Arm of Bonne Bay, Newfoundland in December of 2013. During these trials a series of calibration profiles with the mass shifting and ballast mechanisms at different locations were performed before and after the survey portion of the trials. Further trials were performed in the Labrador Sea in July of 2014 with two reduced sets of calibration runs. The nominal ellipsoidal coefficients were extracted using the full set of measurements from a set of calibration profiles and used as the initial conditions for the polynomials which define each parameterized coefficient. These polynomials as well as the sensor misalignment matrix were then optimized using a gradient descent solver which minimizes both the total magnetic field difference and the vertical magnetic field variance between the modeled and measured values. Including the vertical field in this manner allows for convergence in spite of severe limitations on the platform's motion and for computation of the vehicle's magnetic heading.
\end{abstract}

\section{Introduction}

The use of underwater vehicles as a platform for oceanic research is an excellent way to collect high quality data in a challenging environment. Long range AUVs, capable of traveling thousands of kilometers before needing to be recovered are recently the focus of significant interest (Furlong et al., 2012). Underwater gliders are a type of long range underwater vehicle, however, they require surface access for navigation, have limited speed and require vertical translation for forward movement (Rudnick et al., 2004). For these vehicles minimizing energy consumption is one of the primary design and operational goals.

The use of magnetic field measurements as a heading reference for navigation in underwater vehicles has been well established (Kinsey et al., 2006). In recent work earth magnetic information has also been suggested 
for possible use in total-field map based relative navigation techniques (Kato and Shigetomi, 2009; Teixeira and Pascoal, 2008). This use of magnetic measurements for online navigational aiding is the motivation for this research. In such a system, magnetic measurements are capable of augmenting a terrain relative navigation scheme in regions of low terrain variability or when the terrain is beyond the range of the vehicle's acoustic sensors. However, an online implementation of a magnetic aided navigation system has not been realized. This lack of progress has been limited by the challenges involved in instrumenting and calibrating an underwater vehicle for accurate online magnetic measurements and the lack of suitably high resolution magnetic maps.

Measurements of magnetic fields must be calibrated to remove the effects of the sensing platform and to correct for the non-linearity of the sensor. These effects can be due to electrical currents, hard and soft magnetic effects and temperature variations among others. Recent methods have found success through estimating the parameters through batch estimation in which a series of calibration measurements are performed periodically throughout the measurement regime. Calibration parameters are then extracted using the set of measurements through vector or scalar methods.

Vector calibration methods attempt to preserve the three vector components of the magnetic field measurement in the earth frame (Isezaki, 1986; Korenaga, 1995). In these methods the platform is maneuvered in a figure-eight pattern to provide an unbiased representative sample. The measurements are subsequently rotated to the earth frame and the calibration coefficients are extracted through a least squares fit to the data. The drawback of these methods is the requirement for a precise attitude reference such as a laser ring gyro or similar system which can be cost prohibitive both monetarily and in energy consumption.

Scalar methods, on the other hand, make use of only the magnitude of the magnetic field to compute the calibration coefficients resulting in the vector components of the magnetic field remaining in the sensor frame. Initial developments of batch scalar calibration methods of vector magnetometers in a laboratory setting were performed for the satellite industry (Merayo et al., 2000). These methods were verified through several years of measurements on the Orsted satellite through comparison of the calibrated vector magnetometer measurements with an on-board total field magnetometer (Olsen et al., 2003). Around the same time, a method termed the TWOSTEP algorithm was published for which initially the bias (Alonso and Shuster, 2002a), followed by the complete set of calibration parameters are estimated (Alonso and Shuster, 2002c) which were then refined through a Gauss-Newton iterative solver (Alonso and Shuster, 2002b). These methods saw quick adoption by marine magnetic survey groups with towed marine magnetometer systems calibrated through an initial lab calibration using these methods, followed by further calibration maneuvers in the field to refine the parameters (Gee and Cande, 2002; Engels et al., 2008). Developments of new methods for estimating ellipsoids from point measurements (Turner et al., 1999; Fitzgibbon et al., 1999; Li and Griffiths, 2004; Markovsky et al., 2004) led to a further refinement of the TWOSTEP algorithm to a method based on a least squares estimation of ellipsoidal parameters (Foster and Elkaim, 2008). This least squares method was then extended to a maximum likelihood estimator of the magnetic calibration coefficients (Vasconcelos et al., 2011). Similar developments use an adjusted least squares method that includes a noise term to allow for unbiased estimates of the calibration parameters in sensors with higher noise floors (Renaudin et al., 2010a; Renaudin et al., 2010b).

An extension to batch estimation has been proposed in which an initial estimate of the calibration parameters is obtained followed by real time adaptation of the parameters through non-linear estimators (Crassidis et al., 2005). Further extensions have shown through off-line analysis compensation methods for a time varying bias due to onboard electrical currents (Springmann, 2013), the presence of actuators with moving magnetic parts (Frey et al., 2014) and for a varying sensor altitude during the calibration run (Bronner et al., 2013).

Of these, vector calibration methods are more suited to vehicles which have limitations in their controllable degrees of freedom such as an underwater glider. However, they require a precision attitude reference to rotate the magnetic measurements to the horizontal plane which is infeasible on an underwater glider due to their relatively large energy consumption. Additionally, long range underwater vehicles, and underwater gliders in particular, require additional effort to calibrate the magnetic field measurements. This extra effort 
is due to the use of an adjustable internal battery shifting mechanism which translates a battery assembly to achieve attitude control and therefore includes hard and soft magnetic materials.

As a step towards a magnetic anomaly relative navigation system this work examines suitable methods for calibrating, instrumenting, performing magnetic measurements with an underwater glider and comparing those measurements to a prior digital model of the magnetic anomaly field. The variable locations of the battery shifting and ballast mechanisms on the underwater glider provide an additional challenge for calibrating the magnetic measurement system which is untreated in the prior literature. As such, a parameterized field calibration method is presented which fits polynomial functions to the calibration parameters based on the actuator locations. To this end the theory for a nominal geometric calibration and a parameterized geometric calibration method is presented and the underwater glider equipped with the magnetic instrumentation developed for this work is introduced. The calibration procedures are demonstrated on field data gathered using the underwater glider during trials in the East Arm of Bonne Bay as well as in the Labrador Sea. The calibrated data are compared with magnetic anomaly models produced from prior aeromagnetic surveys of the region. The use of the calibrated measurements as a heading reference is also examined.

\section{Calibration Methods}

Measurements of the earth's magnetic field must be calibrated in order to remove the effects of the sensing platform. These effects can be due to instrument non-linearities as well as hard and soft magnetic effects.

\subsection{Nominal Geometric Calibration}

An ideal magnetic sensor at a fixed location produces measurements with a constant magnitude resulting in the data lying on the surface of a sphere, centered on the origin with the radius equal to this magnitude. Distortions due to the sensor errors and the vehicle hard and soft magnetic effects have been shown to cause the measurements to be translated, rotated and scaled such that the sphere becomes an ellipsoid.

If the movable masses in the vehicle are held stationary the hard and soft magnetic effects from the vehicle as well as scaling, bias and other instrument errors may be calibrated for using geometric batch methods (Vasconcelos et al., 2011; Bronner et al., 2013; Renaudin et al., 2010a). These methods assume a constant magnetic field and rely on rotations of the instrument through the calibration space such that an ellipsoid may be fit to the distorted data. Using these ellipsoid translation, rotation and scaling coefficients the distorted data may be corrected back to a sphere, centered on the origin with a magnitude equal to the local total field.

The problem of finding this set of translation, rotation and scaling coefficients can be expressed in matrix notation as

$$
[\mathbf{R}, \mathbf{S}, \mathbf{T}]=G\left(\mathbf{H}_{r}\right)
$$

where $\mathbf{R}, \mathbf{S}$, and $\mathbf{T}$ are the rotation, scaling and translation matrices that are representative of the ellipsoidal fit $G()$ to the raw magnetic data $\mathbf{H}_{r}$. Geometrically, the translation coefficients are the distance from the center of the ellipsoid to the origin, the scaling coefficients are the magnitudes of the major and minor ellipsoid axes and the rotation coefficients are the rotations of the major and minor axes of the ellipsoid. The ellipsoid equation representing the relationship between the raw magnetic data and the corrected data is written as

$$
\mathbf{H}_{r}=H_{e}^{-1} \mathbf{S R H}_{c}+\mathbf{T}
$$

where $\mathbf{H}_{c}$ is the calibrated magnetic data in the sensor frame and $H_{e}$ is the magnitude of the local magnetic field at the calibration location. The raw magnetic data may then be translated, rotated and scaled accordingly by re-arranging the ellipsoid equation to

$$
\mathbf{H}_{c}=H_{e} \mathbf{S}^{-1} \mathbf{R}^{-1}\left(\mathbf{H}_{r}-\mathbf{T}\right)
$$


This calibration procedure normalizes the magnitude of the magnetic measurements due to the product of the inverse of the scaling coefficients. To give the calibrated values units, the normalized values must be scaled by $H_{e}$ which often may be approximated from the International Geomagnetic Reference Field (IGRF) (International Association of Geomagnetism and Aeronomy, Working Group V-MOD. Participating members et al., 2010). However, the IGRF does not include many of the higher frequency components and the local magnetic anomalies. A higher resolution model or some other independent measurement at the calibration location are therefore preferred. If a local anomaly map is available these anomaly values may be included as in

$$
H_{e}=\left\|\mathbf{H}_{I G R F}\right\|+H_{a}
$$

where $H_{a}$ is the magnitude of the magnetic anomalies at the calibration locations. The resulting values given by $\mathbf{H}_{c}$ are the calibrated measurements of the magnetic field in the sensor frame for a vehicle with fixed locations of the hard and soft magnetic influences and no significant electrical currents.

\subsection{Parameterized Geometric Calibration}

For vehicles with moving hard or soft magnetic parts that have a number of steady state values a parameterized version of the geometric calibration method is proposed. In this method the nominal geometric calibration procedure from section 2.1 is performed on data gathered from a number of different steady state values for each of the moving parts. The global calibration parameters are used as the initial conditions for an iterative gradient decent solver which optimizes third order polynomial functions for each of the calibration parameters with each of the moving masses as parameters. In the case of underwater gliders, the primary parameters are the moving mass mechanism used for fine control of the vehicle pitch and the ballast mechanism which is responsible for the large pitch and buoyancy changes between diving and climbing. The geometric fitting then becomes of the form

$$
[\mathbf{R}, \mathbf{S}, \mathbf{T}]\left(p_{m}, p_{b}\right)=G\left(\mathbf{H}_{r}\left(p_{m}, p_{b}\right)\right)
$$

where each of the rotation, translation and scaling coefficients are a function of the moving mass location $p_{m}$ and the ballast piston location $p_{b}$. The parameterized ellipsoid equation is similarly given as

$$
\mathbf{H}_{r}=H_{e}^{-1} \mathbf{S}\left(p_{m}, p_{b}\right) \mathbf{R}\left(p_{m}, p_{b}\right) \mathbf{H}_{c}+\mathbf{T}\left(p_{m}, p_{b}\right)
$$

Upon re-arranging, the raw magnetic data may be corrected by computing the translation, rotation and scaling matrices for a given moving mass and ballast location as in

$$
\mathbf{H}_{c}=H_{e} \mathbf{S}\left(p_{m}, p_{b}\right)^{-1} \mathbf{R}\left(p_{m}, p_{b}\right)^{-1}\left(\mathbf{H}_{r}-\mathbf{T}\left(p_{m}, p_{b}\right)\right)
$$

The parameterized functions are found by assigning polynomials to the set of individual calibration coefficients. These polynomial functions are of third order and take the form of

$$
\begin{array}{r}
c_{0} p_{m}^{3}+c_{1} p_{b}^{3}+c_{2} p_{m}^{2}+c_{3} p_{b}^{2}+c_{4} p_{m}^{2} p_{b}+c_{5} p_{m} p_{b}^{2} \\
+c_{6} p_{m} p_{b}+c_{7} p_{m}+c_{8} p_{b}+c_{9}
\end{array}
$$

resulting in a total of 90 coefficients required for the two parameter calibration problem. These parameters may be found using a gradient descent solver which minimizes error between the local field and the magnitude of the calibrated measurements as in

$$
\min \left(\left\|\mathbf{H}_{c}\right\|-H_{e}\right)
$$

As an additional constraint the variance of the difference between the vertical component of the calibrated measurements in the horizontal plane and the vertical component of the IGRF at the calibration location may be minimized as in

$$
\min \left(\operatorname{var}\left(H_{h z}-H_{I G R F z}\right)\right)
$$

This constraint is important to include for systems with limitations in their controllable range of motions as it restricts the rotations of the calibration ellipsoid to sensible values. Additionally, by adding this constraint 
the sensor misalignment terms may be added to the solver as scalar coefficients increasing the degrees of freedom of the solver to 93. However, since this constraint is only used in the solver the noise from the attitude sensor does not pass to the calibrated total magnetic field measurements in the sensor frame.

In this solver every parameter is perturbed by a positive and negative amount with the initial conditions given by the global calibration coefficients which are assigned to the $c_{9}$ polynomial terms and assuming zero sensor misalignment. The perturbation with the minimum error selects the direction, which the solver continues along while the error continues to decrease. When this condition fails the cycle repeats with a new direction selected. This solver continues until the incremental difference in the error reaches a suitably small threshold.

While scalar measurements of the magnetic field are useful for geophysical models and interpreting geological features, vector measurements in the horizontal plane are useful as a heading reference. To bring the measurements to the horizontal inertial plane the calibrated measurements are rotated to the vehicle frame by the sensor misalignment matrix $\mathbf{R}_{s}$ and subsequently to the horizontal plane using the vehicle rotation matrix $\mathbf{R}_{v}$ as in

$$
\mathbf{H}_{h}=\mathbf{R}_{v} \mathbf{R}_{s} \mathbf{H}_{c}
$$

where $\mathbf{H}_{h}$ are the calibrated magnetic measurements rotated to the horizontal plane. In this case the vehicle rotation matrix is composed of the vehicle pitch and roll as measured by the attitude sensor. The vehicle's heading $\beta$ may then be estimated as

$$
\beta=\operatorname{atan} 2\left(-H_{h y}, H_{h x}\right)
$$

where $H_{h x}$ and $H_{h y}$ are the $\mathrm{x}$ and $\mathrm{y}$ component of the calibrated measurements rotated to the horizontal plane.

\section{Instrumentation}

An underwater glider's energy is provided by on-board batteries which gives it an endurance of around one month when using alkaline primary cells and six months when using lithium primary cells. In a standard configuration of a vehicle equipped only with a conductivity, temperature and pressure sensor (CTD), the vehicle uses an average power of around one Watt. To not significantly impact the endurance or range of the vehicle, additional sensors should use as little power as possible. Therefore, to instrument an underwater glider with a precision magnetic sensor, the power consumption of the device must remain low to minimize the impact on the vehicle's endurance.

While progress is being made towards lower power cesium vapor magnetometers which would be well suited to integration in mobile platforms, the power consumption of presently available devices still remains on the order of Watts (Shah et al., 2007; Prouty and Johnson, ). Fluxgate sensors, on the other hand, have power requirements down to the level of 10s of milliwatts. For this reason the chosen sensor is a low power tri-axial Mag-648 fluxgate magnetometer by Bartington Instruments which consumes around 14 milliwatts (Bartington Instruments, 2011). Low power fluxgates of this type are often subject to higher degrees of noise, orthogonality errors, and offset errors than higher power versions (Primdahl, 1979). While the impact of the higher noise is mitigated through low frequency sampling requirements and filtering which reduce the bandwidth of these sensors, the orthogonality errors and offset errors require careful calibration. Additionally, the offset error settles to a slightly different value each time the sensor is powered on requiring the sensor to remain energized once calibrated.

The fluxgate sensor is mounted in a strap-down configuration in the vehicle's payload bay. The device is powered by a set of independent batteries and is sampled using an isolated 24-bit sigma-delta analog to digital converter (ADC). This ADC uses several different internal low pass filters and modifies the filter coefficients based on the sampling rate selected. The effective resolution of the device is therefore variable with the sampling rate. The inputs to the ADC have anti-aliasing filters with a corner frequency of $0.33 \mathrm{~Hz}$ 
to mitigate high frequency noise from the electronics and other systems. The ADC uses the serial peripheral interface (SPI) to send the data to the glider payload computer where it is logged at a frequency of $0.25 \mathrm{~Hz}$. The ADC used has a single digitizer and samples of each channel are taken at different times requiring the time stamp of each channel's measurement to be recorded such that the measurements may be interpolated to the same time base. While keeping the noise floor low overall this sequential and slow digitizing scheme has the drawback of lumping noise from spurious electrical currents into the measurements. Additionally, sensor rotations which cause large changes in signal are subject to phase lag due to the heavy analog filtering done to the signals prior to sampling.

The electrical current drawn by the fluxgate and its electronics is around $4.5 \mathrm{~mA}$. As a result of this low energy consumption, a single set of three AA alkaline cells connected in series will power the fluxgate and its electronics for one month. The goal of not influencing the endurance of the underwater glider while staying within the size and weight requirements for the payload are therefore achieved.

\section{$4 \quad$ Field Trials}

Two sets of field trials using the magnetic fluxgate sensor installed on the 200 meter Slocum Electric glider were performed to evaluate the efficacy of making magnetic measurements using this platform. The initial trials were performed to evaluate the parameterized calibration method using a set of five different battery locations during descending and ascending spirals. While these trials demonstrated the utility of the method, the proximity to coastal magnetic influences made it desirable to perform further open ocean tests. Additionally, the initial set of trials took nearly six hours to perform, cutting significantly into the range of the vehicle if a calibration routine were performed regularly over the course of a month long deployment. As such the open ocean calibration runs were reduced to a single mass shifting location during ascending and descending spirals. The initial set of parameterized calibration method trials took place in December, 2013 in the East Arm of Bonne Bay, Newfoundland. The field trials with only a single mass shifting location took place in the Labrador Sea in July, 2014.

\subsection{Coastal Trials in Bonne Bay}

The first set of parameterized calibration field trials took place in December, 2013 in the East Arm of Bonne Bay, Newfoundland. In these trials the underwater glider was launched from the small aluminum boat Freezy and after launch was controlled from the Bonne Bay Marine Station.

During the deployment there were light winds and the air temperature was around $-10^{\circ} \mathrm{C}$. Recovery of the vehicle was originally planned for December 12 th but had to be delayed due to strong winds. The vehicle was left to loiter in the lee of the head on Norris Point until a lull in the winds on the 13th allowed the recovery of the vehicle.

After the deployment, a series of clockwise calibration spirals were performed with the vehicle commanded to set the movable battery once during each ascent or descent to achieve a certain pitch according to a look up table. In this way five different battery locations were tested for two different ballast conditions. The ballast was also set to a single value, once for each ascent or descent. Each calibration run therefore consisted of a single spiraling descent and ascent with the ballast and battery at a fixed location and took around 30 minutes to complete.

Another full calibration procedure was repeated prior to recovery. The calibration runs are summarized in Table 1.

The vehicle was then flown in a criss-cross pattern down into the bay and back again with a commanded pitch of plus or minus 26 degrees and a commanded ballast of plus or minus $200 \mathrm{~cm}^{3}$. During this survey 
Table 1: Calibration runs for the parameterized magnetic calibration trials

\begin{tabular}{|l|l|l|l|l|l|}
\hline Run & Direction & $\begin{array}{l}p_{b} \\
{\left[\mathrm{~cm}^{3}\right]}\end{array}$ & $\begin{array}{l}\text { Pitch } \\
{[\mathrm{deg}]}\end{array}$ & $\begin{array}{l}p_{m} \text { Trial 1 } \\
{[\mathrm{in}]}\end{array}$ & $\begin{array}{l}p_{m} \text { Trial 2 } \\
{[\mathrm{in}]}\end{array}$ \\
\hline 1 & Dive & -200 & -14 & 0.272 & 0.226 \\
\hline 2 & Climb & 200 & 14 & -0.181 & -0.139 \\
\hline 3 & Dive & -200 & -18 & 0.380 & 0.274 \\
\hline 4 & Climb & 200 & 18 & -0.234 & -0.191 \\
\hline 5 & Dive & -200 & -22 & 0.428 & 0.375 \\
\hline 6 & Climb & 200 & 22 & -0.289 & -0.246 \\
\hline 7 & Dive & -200 & -26 & 0.491 & 0.400 \\
\hline 8 & Climb & 200 & 26 & -0.344 & -0.300 \\
\hline 9 & Dive & -200 & -30 & 0.527 & 0.472 \\
\hline 10 & Climb & 200 & 30 & -0.401 & -0.348 \\
\hline
\end{tabular}

portion of the mission the vehicle performed profiles down to the vehicle's maximum operating depth of 200 meters where possible, using its altimeter to stay a safe distance, around 10 meters, from the seafloor in shallower regions. The calibration locations and vehicle track-line are shown against the residual magnetic field and bathymetry (Tittensor, 2003) in Fig. 1.

To provide reference measurements, aeromagnetic data overlapping the East Arm of Bonne Bay was used from the Newfoundland and Labrador Geoscience Atlas (Honarvar et al., 2013). Unfortunately, the East Arm is split in half by the boundary of two different high resolution aeromagnetic surveys, the 2009 Corner Brook survey and the 2012 Offshore Western Newfoundland survey. To obtain a reference grid both residual magnetic grids were upward continued to a constant altitude of 90 meters. The grids were then combined, using the average value in the regions of overlap. A mask was applied to these larger grids to limit the region to the area of the East Arm of Bonne Bay. To smooth any discontinuities, 20 passes of a $3 \times 3$ Convolution (Hanning) filter were applied to remove the high frequency content introduced by combining the grids.

For the parameterized calibration method, an initial global fit of the nominal geometric method was performed by using the full set of raw measurements from each of the calibration runs. The extraction of the magnetic calibration parameters using ellipsoid fit methods generally requires adequate coverage of the calibration space such that the ellipsoid is constrained. A single descending or ascending spiral results in a single circle in the calibration space which is insufficient to constrain the ellipsoid. To deal with this sparsity, the initial global fit was performed by using this full set of raw measurements from the diving and climbing calibration runs. In this case the calibration data appears as two circles representing the diving and climbing spirals as shown in Fig. 2.

To constrain the ellipsoid in this initial fit it was necessary to make the $\mathrm{x}$ and $\mathrm{z}$ scaling values equal as there were no calibration measurements in the "northern hemisphere" of the calibration space. Further, the ellipsoid was constrained in rotation such that $\mathbf{R}=\mathbf{I}$ to prevent unrealistic rotations. Normally, the rotations in $\mathbf{R}$ account for the orthogonality errors in the instrument. While the Mag648 fluxgate sensor is known to have slight orthogonality errors the coverage of the calibration space as illustrated in Fig. 2 is insufficient to resolve these rotations when using the nominal geometric calibration method (Bartington Instruments, 2011). The resulting magnitude of the calibrated measurements using these global fits are shown against the raw measurements in Fig. 3 with a closer view in Fig. 4. The nominal geometric method results in a root mean square error between the total field estimate from the IGRF and aeromagnetic data and the calibrated data of $166 \mathrm{nT}$ and $147 \mathrm{nT}$ for the first and second set of calibration runs respectively.

The global fit was then used as the initial conditions for the parameterized equations by setting the $c_{9}$ coefficients from Eqn. 8 to be equal to the ellipsoid's scaling, translation and rotation coefficients. The parameterized equations were then adjusted using the gradient descent optimization scheme described earlier. In this optimization scheme the local total field was computed from the IGRF model and the magnetic 

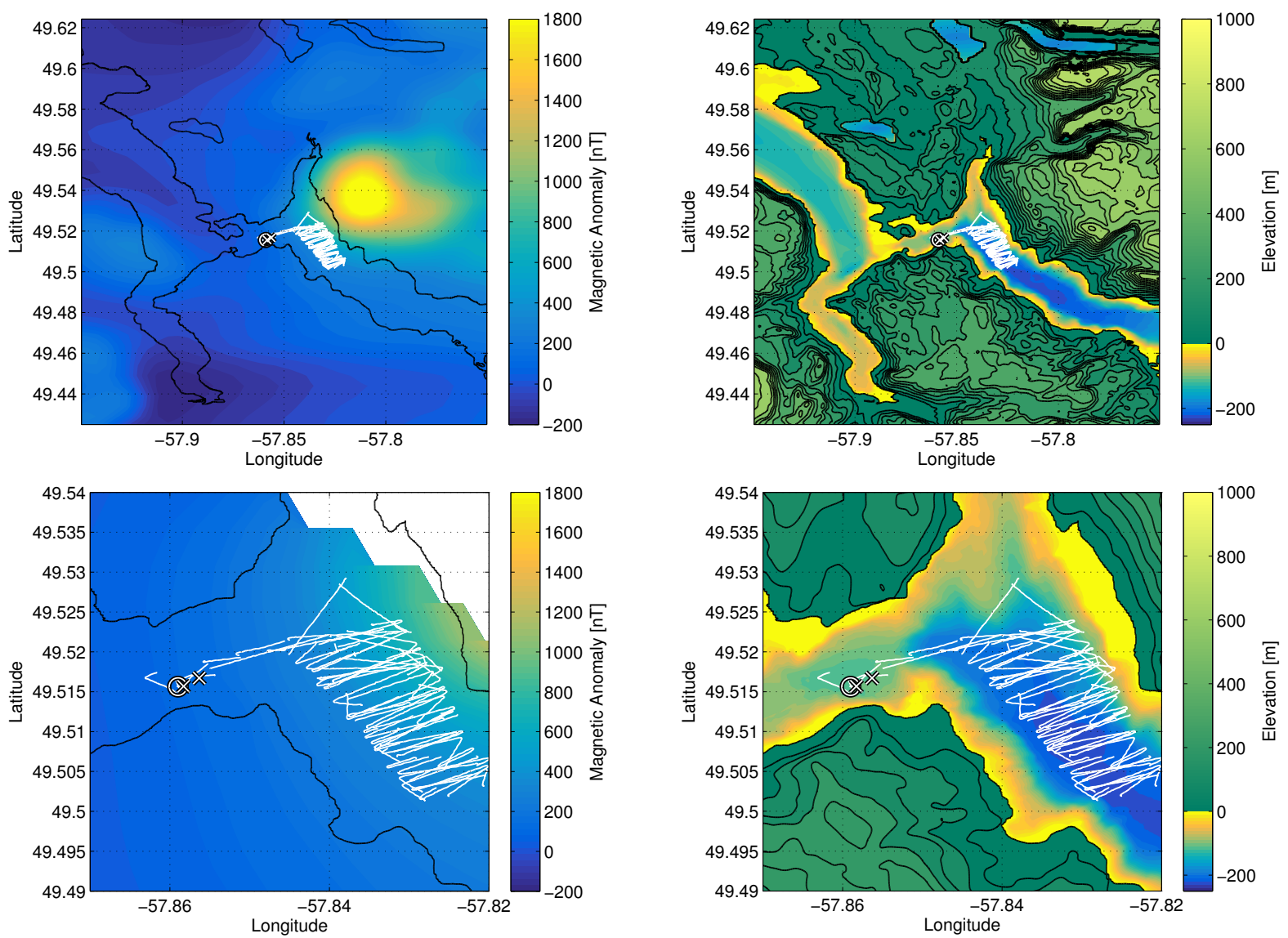

Figure 1: Calibration locations (x's) and the Bonne Bay Trials track-line (white line) starting from the circle and proceeding to crisscross south and then north in the East Arm of Bonne Bay. The magnetic anomalies of the Bonne Bay region from the Magnetic Anomaly Map of North America (Bankey et al., 2002) (top left) with closeup from the 2009 and 2012 aeromagnetic surveys (bottom left) illustrates the large anomaly adjacent to the trials (shoreline in black). The regional geographic setting for the trials (top right) shows the large anomaly to be coincident with the mountain to the east of the bay. The closeup of the geographic setting (bottom right) shows a bathymetry (Tittensor, 2003) with steep walls and depths down to 230 meters. The calibration spirals were performed in roughly 100 meters of water. 


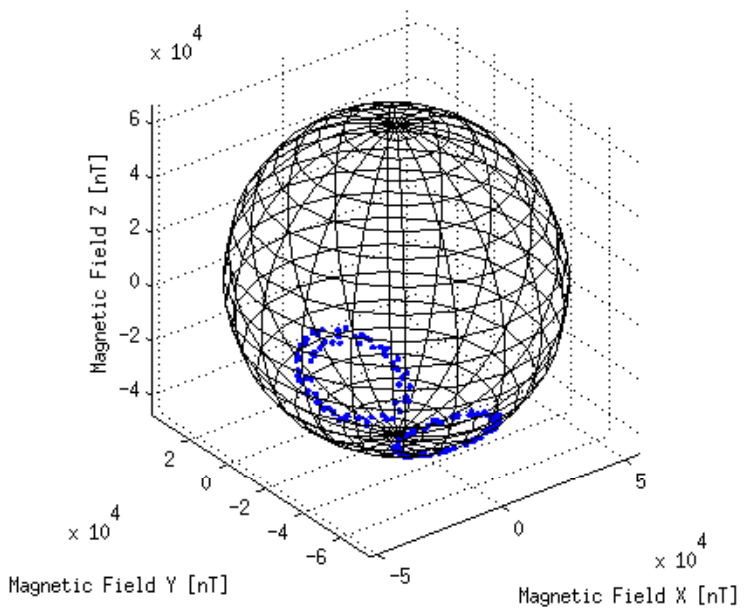

Figure 2: Raw magnetic data from the clockwise and counterclockwise calibration runs over the global calibration ellipsoid
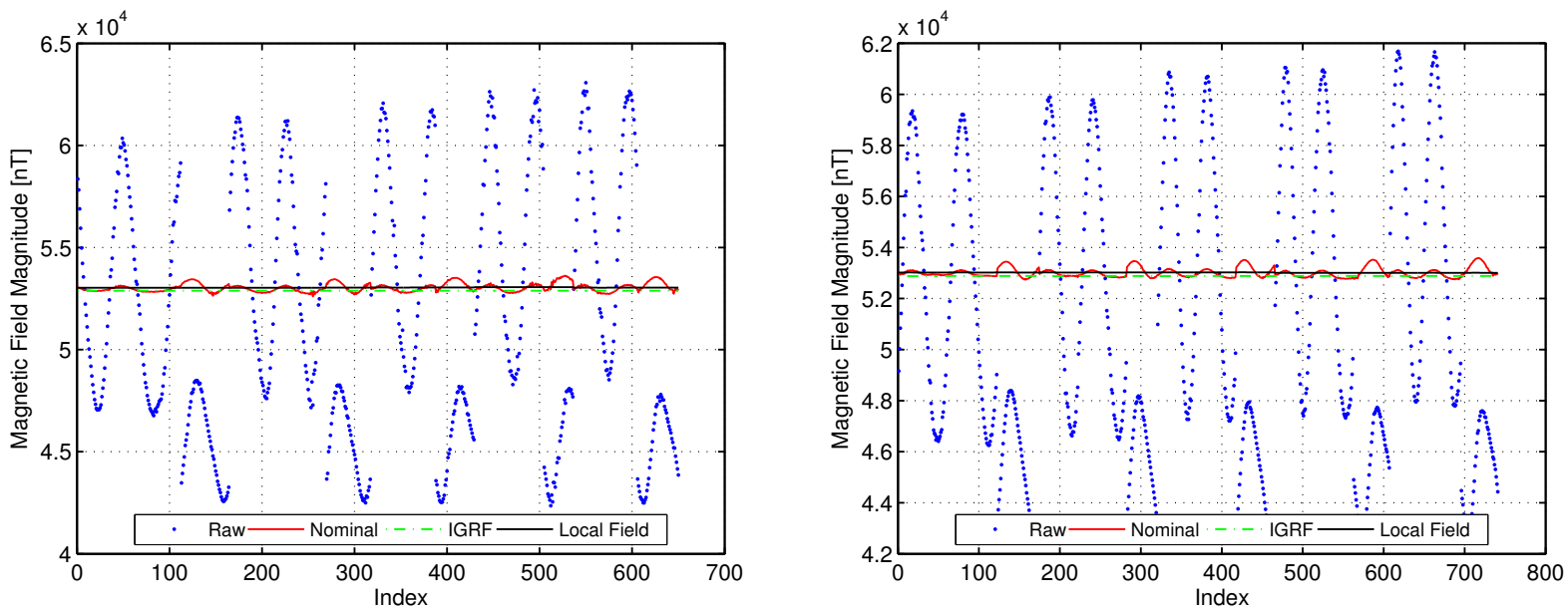

Figure 3: Magnitude of the magnetic data using the nominal calibration method before and after correction shown against the IGRF values for the Bonne Bay field trials using the first (left) and second (right) set of calibration coefficients 
anomaly value at the calibration locations. The resulting magnitude of the calibration measurements, corrected with the parameterized coefficients are shown in Fig. 4. The parameterized geometric method results
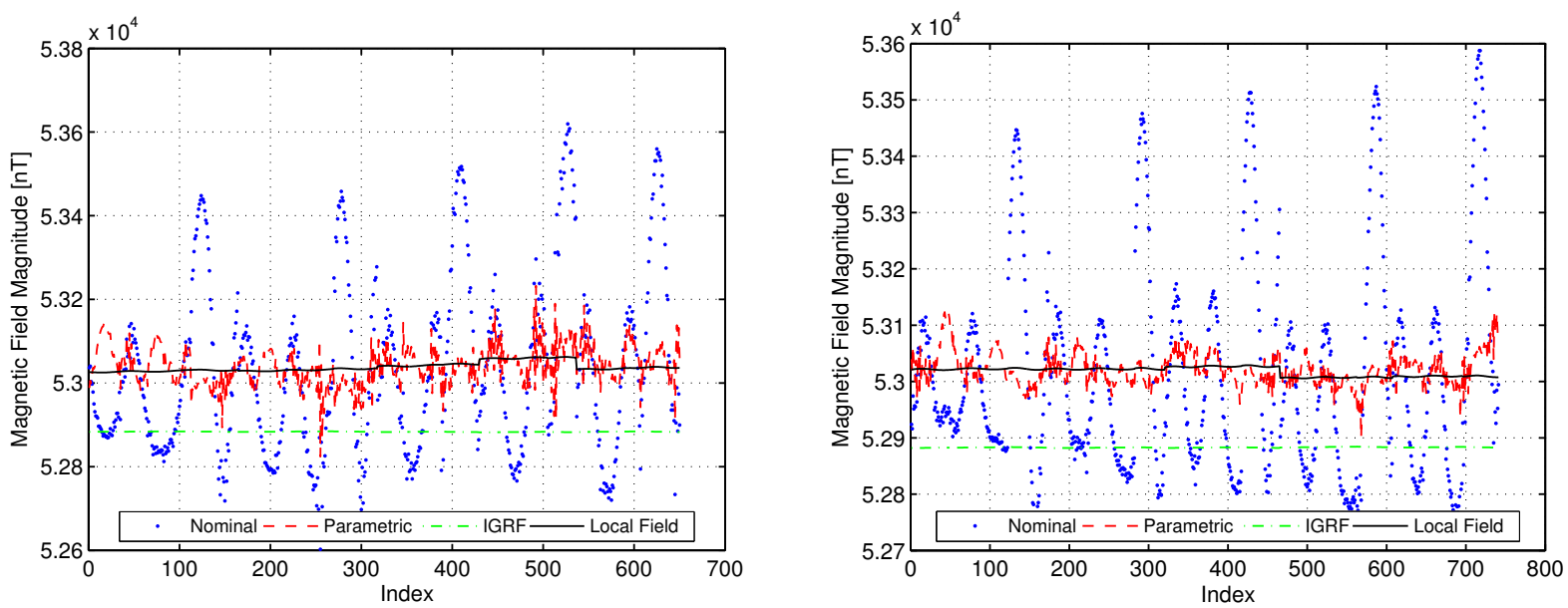

Figure 4: Magnitude of the magnetic data using the nominal and parameterized calibration method with the data from the first (left) and second (right) set of trials shown against the IGRF and local field values for the Bonne Bay trials

in a root mean square error between the total field estimate from the IGRF and aeromagnetic data and the calibrated data of $35 \mathrm{nT}$ and $23 \mathrm{nT}$ for the first and second calibration trials. Additionally, the vertical component of the rotated and calibrated measured vector field, the vertical component of the IGRF field and the difference between the glider heading and the fluxgate heading are shown in Fig. 5.
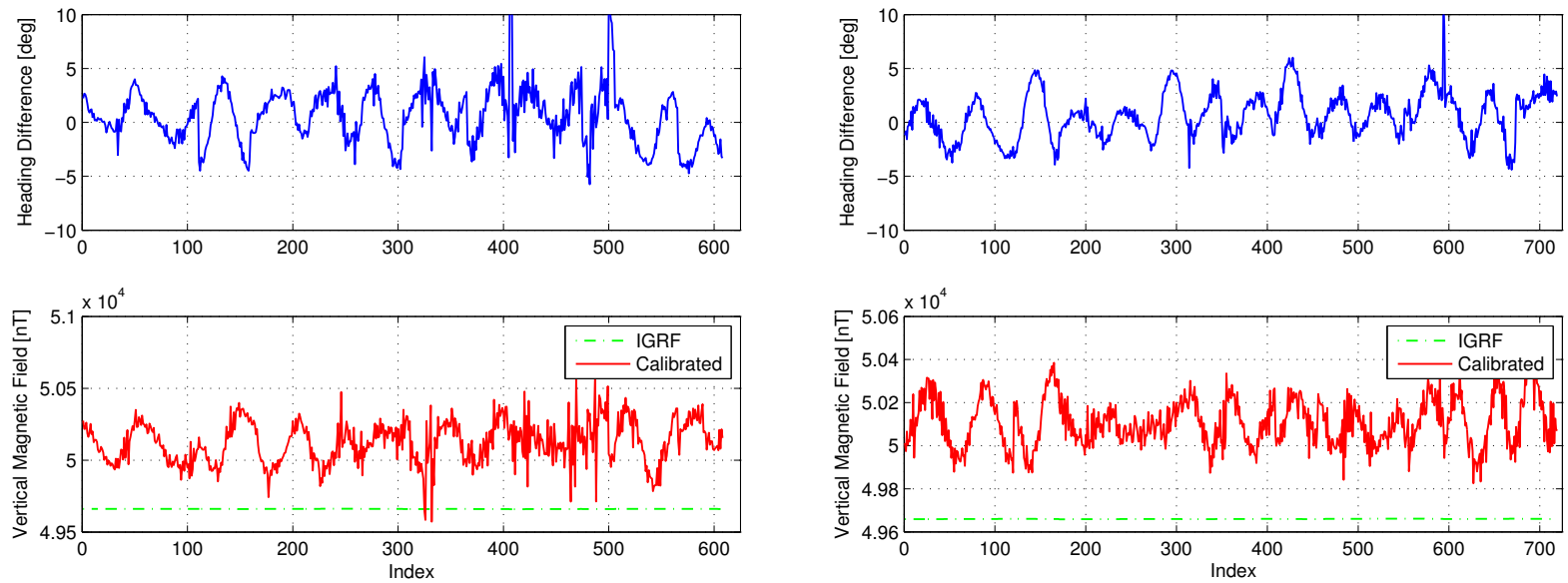

Figure 5: Difference between the glider heading and the fluxgate derived heading (top) for the first (left) and second (right) set of Bonne Bay trials and the vertical component of the IGRF against the measured vertical component by the fluxgate (bottom)

The fluxgate and glider heading have a mean and RMS difference of roughly 0.5 and 2.5 degrees respectively which varies in a somewhat sinusoidal pattern reflective of the spiraling motion during the calibration maneuvers. The mean difference is attributed to sensor misalignment between the glider's heading reference and the fluxgate sensor. The vertical component measured by the fluxgate is expected to have some local offset from the IGRF due to local magnetic anomalies but be relatively constant over the calibration trajectory. In practice there are some differences in the vertical component of the field as the vehicle trajectories have a radius on the order of 10 s of meters and the calibration spirals are performed in a region with significant horizontal gradients in the magnetic anomaly field. 
Each of these sets of parameters is then used to correct the magnetic data gathered during the remainder of the deployment as shown in Fig. 6 .
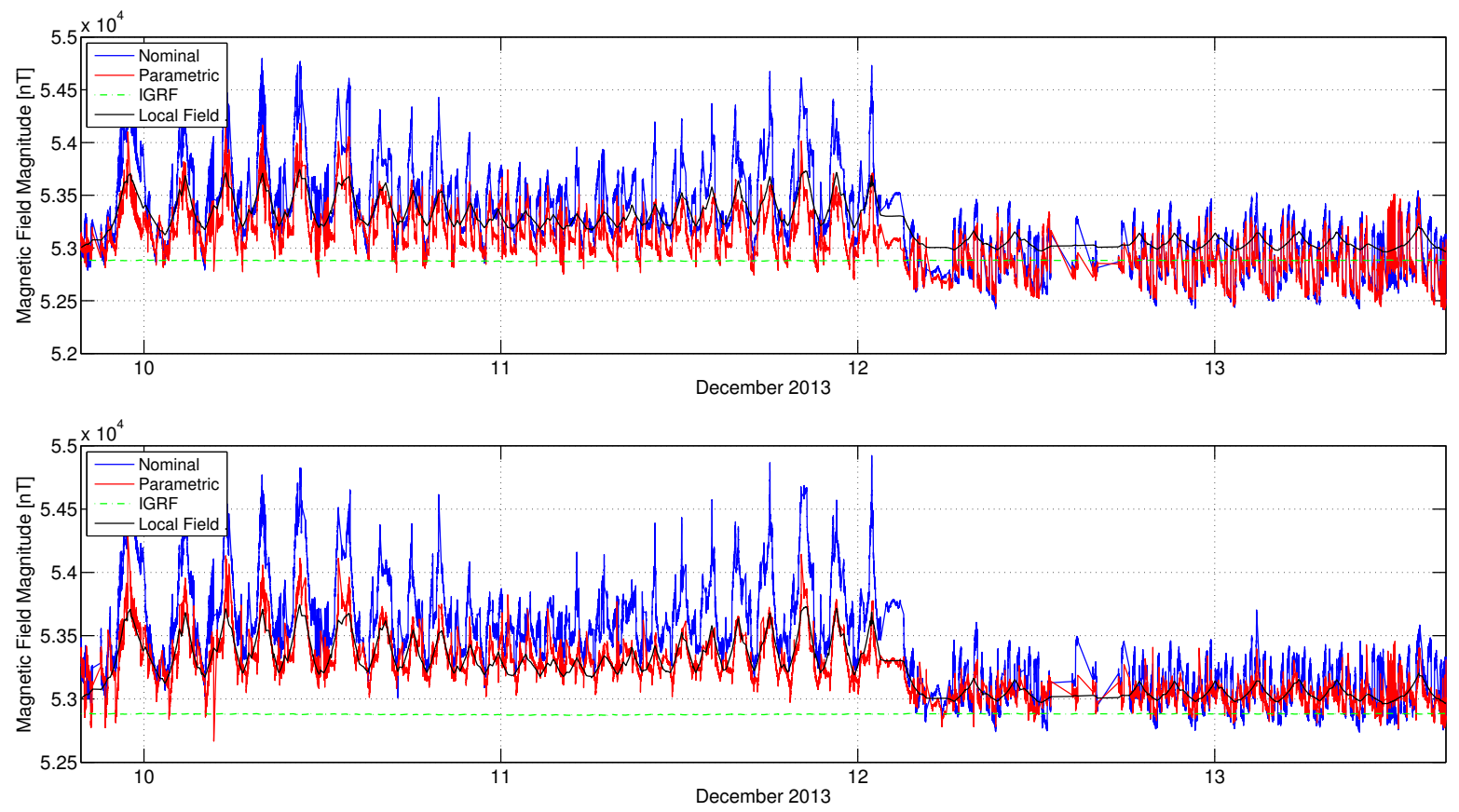

Figure 6: Magnetic data collected during the Bonne Bay deployment in December 2013 shown against the IGRF and local field values calibrated using the first (top) and second (bottom) set of nominal and parameterized calibration coefficients

The calibrated magnetic measurements gathered by the glider may then be compared to the magnetic anomaly gridded data. The measured values contain additional high frequency components not present in the values obtained from the aeromagnetic grids. Some of these differences may be attributed to the aeromagnetic data being collected at a higher altitude reducing the high frequency signatures present in the reference data, the significant low-pass filtering applied during the gridding operations. However, it is possible that the low bandwidth of the fluxgate sensor, at $10 \mathrm{~Hz}$, as well as the sequential sampling of each axis, at $1 \mathrm{~Hz}$ per axis, also contribute to these differences by aliasing spurious signals from electronic or environmental noise into the measurements.

The measured magnetic anomaly data calibrated using the second set of parameterized calibration coefficients shows the closest agreement with the magnetic anomaly data from the aeromagnetic surveys with RMS errors indicated in Table 2. During these trials the raw RMS error between the model and the uncalibrated data over the entire deployment was $4592 \mathrm{nT}$. The parameterized geometric calibration method improves significantly upon this error and provides a modest improvement over the nominal geometric calibration method as well.

Table 2: The RMS errors between the magnetic anomaly map values and the calibrated measurements using the first and second set of nominal and parametric calibration coefficients during the Bonne Bay field trials.

\begin{tabular}{|l|l|l|}
\hline & Nominal & Parametric \\
\hline First & $239 \mathrm{nT}$ & $212 \mathrm{nT}$ \\
\hline Second & $251 \mathrm{nT}$ & $72 \mathrm{nT}$ \\
\hline Both & $218 \mathrm{nT}$ & $115 \mathrm{nT}$ \\
\hline
\end{tabular}

The inability of the parametric method to improve the error using the first set of coefficients is indicative of some unmodeled parameter being dominant. The first set of calibration measurements were gathered 
just after deployment before the temperature of the vehicle normalized to the water temperature. Prior to deployment the vehicle had normalized to the air temperature of $-10^{\circ} \mathrm{C}$. The first set of calibration runs would have been performed while the vehicle was acclimating to the water temperature of $4^{\circ} \mathrm{C}$. The second set of calibration runs was performed after the vehicle had been in the water for several days and would have had ample time to have the temperature equilibrate. The fluxgate sensor is known have a temperature dependency in its offset of $1 \mathrm{nT} /{ }^{\circ} \mathrm{C}$ and scaling of $20 \mathrm{nT} /{ }^{\circ} \mathrm{C}$ which could have resulted in the poor performance of the calibration method in the first set of calibration measurements(Bartington Instruments, 2011). Alternatively, this effect could be caused by differences in the local field at the calibration locations which was not represented by the reference model.

The results using the second set of parametric calibration coefficients indicate that the parameterized calibration method is effective for calibration of magnetic measurements performed from a vehicle with moving masses. The drawback of this method are the increased number of calibration runs that need to be performed over the nominal calibration method.

The Bonne Bay region was initially selected due to the overlap of a high resolution aeromagnetic survey and a body of water with sufficient depth to operate the glider. However, the presence of the large anomaly adjacent to the testing location in the nearby mountain creates several challenges for comparing data collected by the glider in the East Arm of Bonne Bay with prior aeromagnetic survey's. First the aeromagnetic surveys used a draped surface for gathering the data but in areas of high relief, such as the East Arm, this surface can vary significantly from a constant altitude. Additionally, the mountains around the East Arm are an area of strong magnetic features with many sources in the mountains themselves. These features create a challenging environment for interpreting magnetic measurements when some of the sources are above the measurement locations and some are below. The end result of these environmental challenges is that the leveling techniques commonly used to compare data collected at various distances to magnetic sources do not function predictably as these techniques require all of the sources to be below the leveling surface. Additionally, the strong anomalies of the region combined with a profiling vehicle which traverses the entirety of the water column to 10 meters or so from the seafloor makes separation of the various errors difficult. For this reason a second set of trials were performed in the open ocean where all of the sources would be well below the measurement locations and calibrations could occur in deep water.

\subsection{Open Ocean Field Trials in the Labrador Sea}

The open ocean field trials in the Labrador Sea took place from July 4 th to the 17th, 2014. The same underwater glider equipped with the fluxgate sensor was launched from the Canadian Coast Guard Ship Hudson's fast rescue craft as part of the larger Ventilation, Interactions and Transports Across the Labrador Sea project. The glider for this mission had lithium batteries installed to extend its endurance instead of the alkaline set used in Bonne Bay. In total, 13 days of data were collected for a total linear distance of around 250 kilometers. On July 17 the vehicle reported a leak, cutting short the mission and requiring the recovery of the platform.

To reduce the time spent in calibration spirals only a single mass shifting location was used during a set of descending and ascending calibration spirals. During these spirals, as well as the primary mission script, the vehicle was programmed to a fixed battery shifter location gathered from the initial test profile during launch as shown in Table 3.

The battery locations resulting in nominally 26 degree profiles are both set forward of the neutral location due to the mass distribution in the vehicle, suggesting the vehicle is heavy in the tail. The range of the battery shifting mechanism's values from the dive to climb is only $1.58 \mathrm{~mm}$. In the Labrador Sea mission this range was within the deadband of the battery shifting mechanism controller. The battery shifting mechanism, therefore, did not activate between the diving and climbing portions of the profile in this mission but the battery does shift slightly. 
Table 3: Calibration runs for the Labrador Sea magnetic calibration trials

\begin{tabular}{|l|l|l|l|l|l|}
\hline Run & Direction & $\begin{array}{l}\text { Ballast } \\
{\left[\mathrm{cm}^{3}\right]}\end{array}$ & Rotation & $\begin{array}{l}\text { Battery } \\
\text { Command } \\
\text { Trial 1 }[\mathrm{mm}]\end{array}$ & $\begin{array}{l}\text { Battery } \\
\text { Command } \\
\text { Trial 2 }[\mathrm{mm}]\end{array}$ \\
\hline 1 & Dive & -200 & CW & 16.25 & 16.25 \\
\hline 2 & Climb & 200 & CW & 17.83 & 17.83 \\
\hline 1 & Dive & -200 & CCW & 16.25 & 16.25 \\
\hline 2 & Climb & 200 & CCW & 17.83 & 17.83 \\
\hline
\end{tabular}

Twice throughout the trials, the first on July 9th and the second on July 17th, a set of clockwise and counterclockwise spiraling profiles were performed. The locations of these calibration spirals against the magnetic anomaly model and bathymetry are shown in Fig. 7.
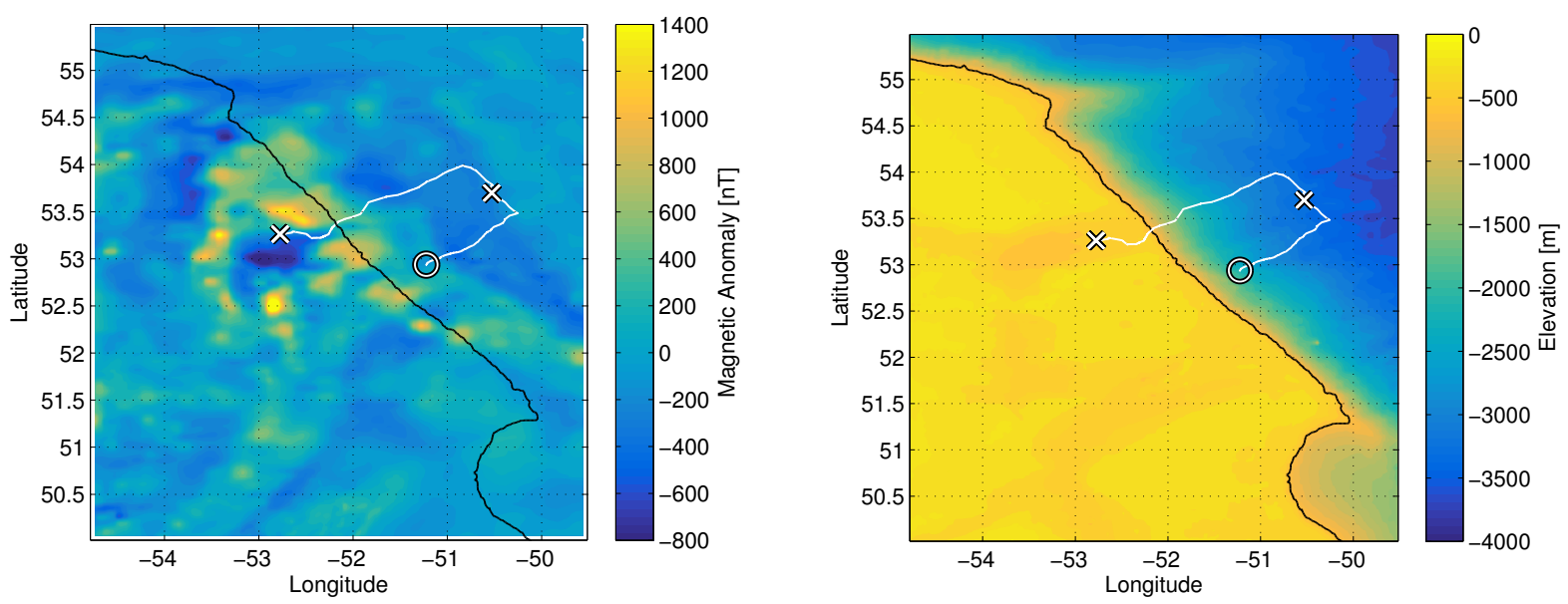

Figure 7: Locations of the calibration runs, indicated by the x's, shown against magnetic anomaly values in nanoteslas from the Magnetic Anomaly Map of North America (left) (Bankey et al., 2002) with the bathymetry from the Global Multi-Resolution Topography database (Ryan et al., 2009) (right). The trajectory of the glider is shown as a white line with the starting location indicated by a circle and the shelf break at 600 meters shown as a black line.

In the Labrador Sea trials the local field is computed from the North American Magnetic Anomaly Map (NAMAM)(Bankey et al., 2002). The NAMAM is compilation from aeromagnetic surveys, marine surveys and is filled in with the 2 arc minute Earth Magnetic Anomaly Grid (EMAG2) which is compiled from satellite measurements (Maus et al., 2009). In the region of the field trials in the Labrador Sea the gridded data in the NAMAM is exclusively from the EMAG2. The bathymetry data was taken from the Global Multi-Resolution Topography database (Ryan et al., 2009).

Similarly to the Bonne Bay trials, global fits were found using the nominal geometric calibration method on each set of data from the first and second set of calibration maneuvers. Prior to calibration the magnetic signal during the calibration data had an RMS error of $7576 \mathrm{nT}$ and $8555 \mathrm{nT}$ respectively as shown in Fig. 8. If the scaling and translation parameters from the initial ellipsoid fit are used to correct the magnetic data from these calibration spirals, with the magnitude $H_{e}$ given by the local field at the calibration location from the NAMAM, an RMS error of $45 \mathrm{nT}$ and $51 \mathrm{nT}$ is achieved as shown in Fig. 9. This nominal fit was then used as the initial conditions for the set of parametric coefficients using the same method described prior. The resulting magnitude of the calibrated measurements, corrected with the parameterized coefficients are also shown in Fig. 9.

The parameterized geometric method results in a root mean square error between the total field estimate 

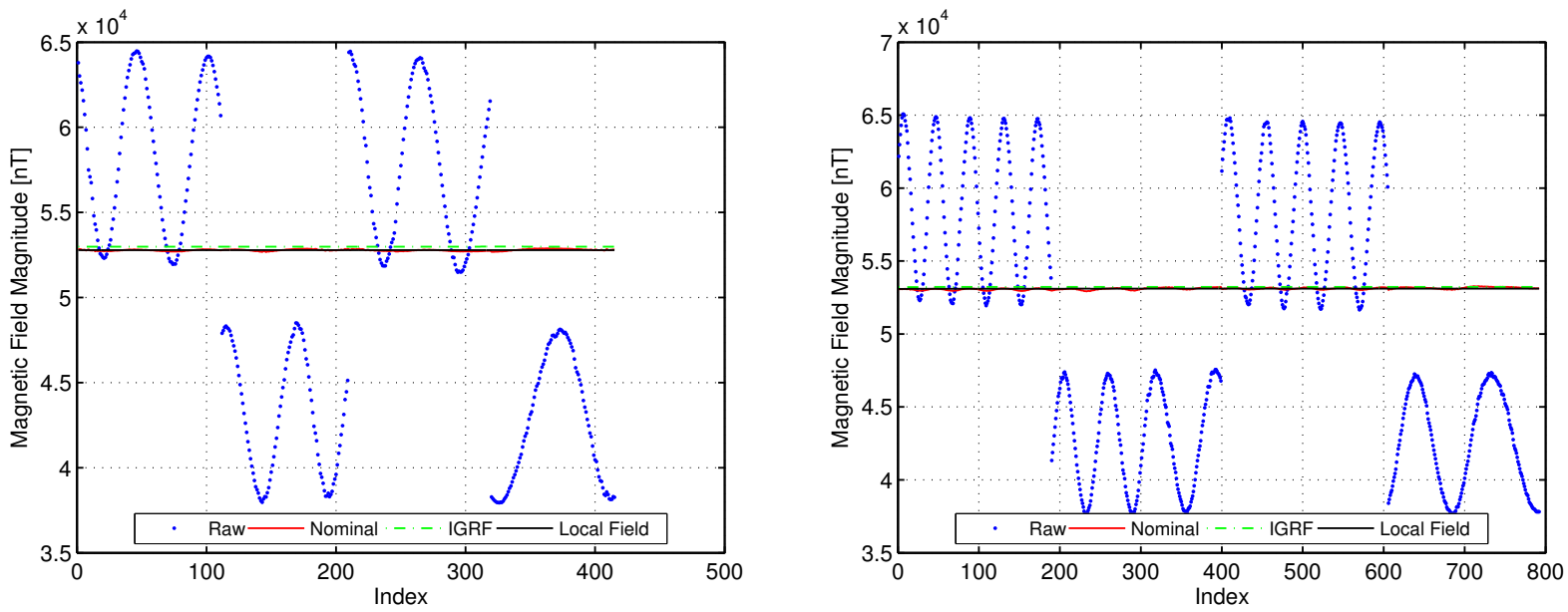

Figure 8: Magnitude of the global calibration spirals before and after calibration shown against the IGRF and the local field values for the Labrador Sea field trials for the first (left) and second (right) calibration where the local field is the IGRF plus the magnetic anomaly values from the North American Magnetic Anomaly Map (Bankey et al., 2002).
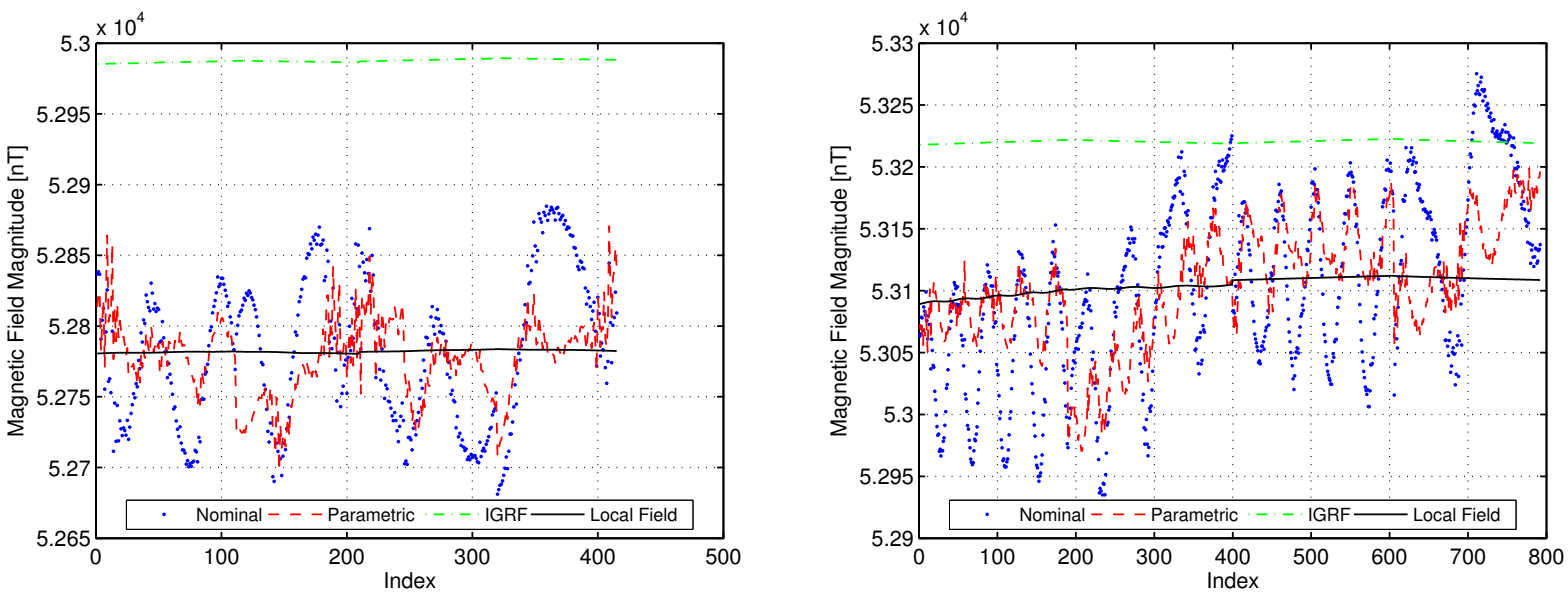

Figure 9: Magnitude of the magnetic data using the nominal and parameterized calibration method with the data from the first (left) and second (right) set of trials shown against the IGRF and local field values for the Labrador Sea trials 
from the IGRF and aeromagnetic data and the calibrated data of $21 \mathrm{nT}$ and $24 \mathrm{nT}$ for the first and second calibration trials in the Labrador Sea. The vertical component of the rotated and calibrated measurements, the vertical component of the IGRF field and the difference between the glider heading and the fluxgate heading are shown in Fig. 10
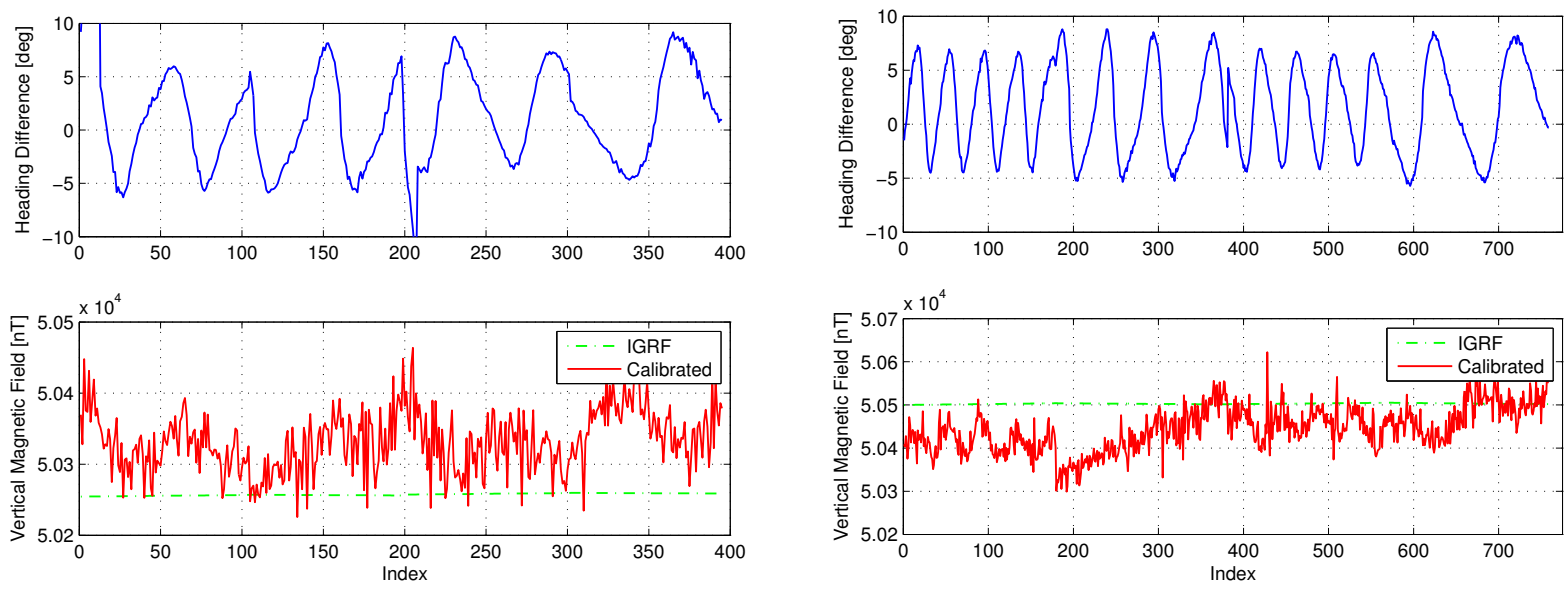

Figure 10: Difference between the glider heading and the fluxgate derived heading (top) for the first (left) and second (right) set of Labrador Sea trials and the vertical component of the IGRF against the measured vertical component by the fluxgate (bottom)

The fluxgate and glider heading differ by a mean of 1.5 degrees and around 5 degrees RMS in a manner similar to that in Bonne Bay trials. The fluxgate sensor had been taken out and re-installed which could account for the difference in mean difference due to sensor misalignment. The larger RMS difference is attributed to the glider's heading reference not being re-calibrated after the alkaline batteries used in the Bonne Bay trials where removed and replaced with the lithium batteries used in the Labrador Sea trials.

Each of these sets of parameters is then used to correct the magnetic data gathered during the remainder of the deployment as shown in Fig. 11. In the Labrador Sea trials, the magnetic field measurements gathered by the underwater glider and calibrated using the first set of calibration coefficients show the closest agreement with the values from the model as in Tab. 4.

Table 4: The RMS errors between the magnetic anomaly map values and the calibrated measurements using the first and second set of calibration coefficients during the Labrador Sea trials.

\begin{tabular}{|l|l|l|}
\hline Trial & Nominal & Parametric \\
\hline First & $222 \mathrm{nT}$ & $61.5 \mathrm{nT}$ \\
\hline Second & $396 \mathrm{nT}$ & $185 \mathrm{nT}$ \\
\hline Both & $291 \mathrm{nT}$ & $69 \mathrm{nT}$ \\
\hline
\end{tabular}

Both of the sets of calibration measurements were performed well after deployment and it is unlikely that the temperature would have such an impact. The location of the second calibration maneuver in the Labrador Sea was in a region that has significant variation in the magnetic anomaly field. This variation is evident in Fig. 9 where the NAMAM shows a change in value of $10 \mathrm{~s}$ of $\mathrm{nT}$. Since the NAMAM is only contains the long wavelength components measured by the satellites it is likely that local field used to calibrate the fluxgate in the second set of trials is inaccurate. This inaccuracy illustrates the need for accurate measurements of the local field to calibrate against. 

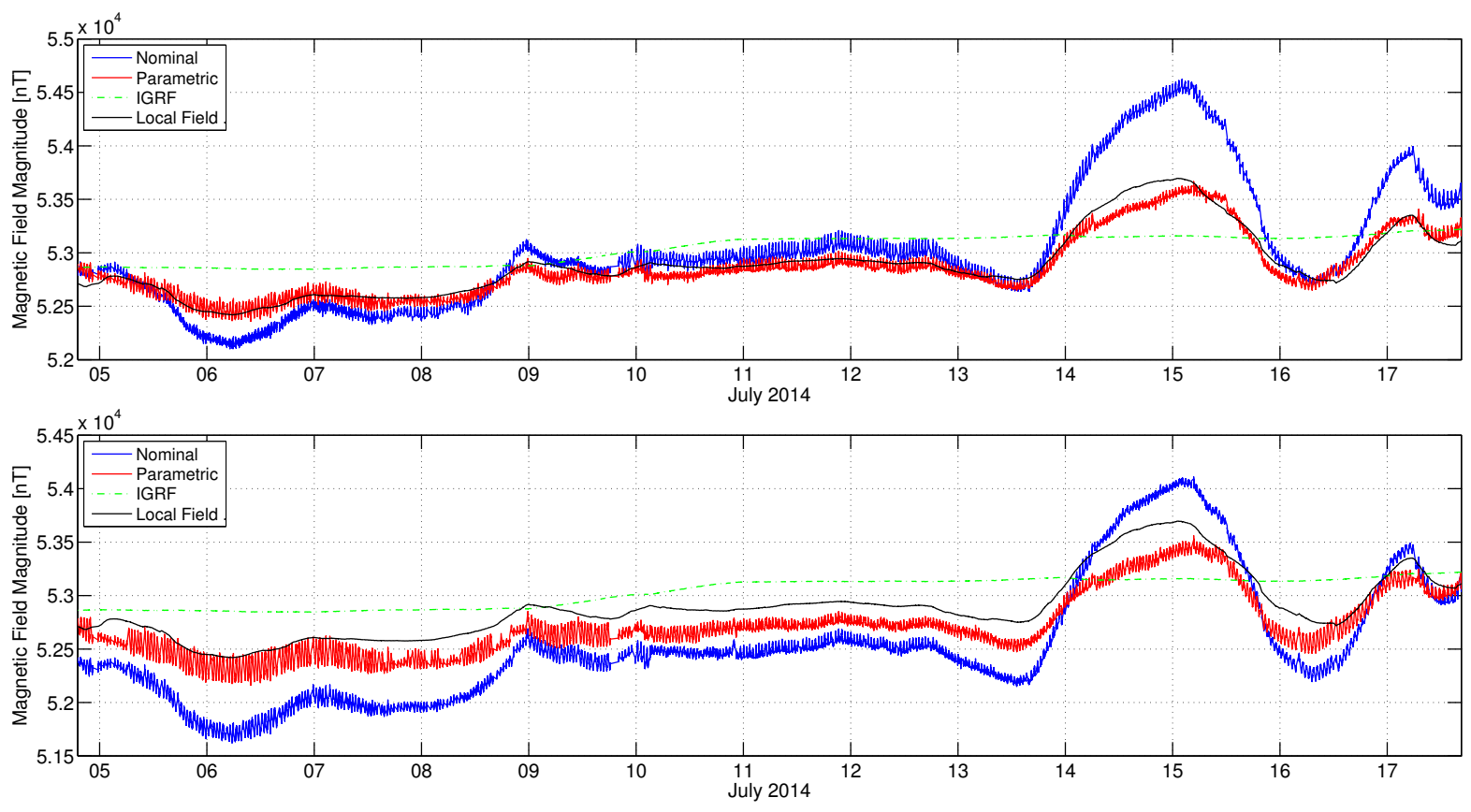

Figure 11: Magnetic data collected during the Labrador Sea Trials shown against the IGRF and local field values calibrated using the first (top) and second (bottom) set of nominal and parameterized calibration coefficients

\section{Conclusions}

Marine observations of the magnetic field gathered by long duration platforms with endurances on the order of months are presently not possible due to calibration limitations. For rigid platforms with fixed components and low levels of electrical noise a geometric calibration method may be used. In this nominal geometric calibration method the raw measurements are assumed to lie on the surface of an ellipsoid. The ellipsoid's offset, radii and rotations of the major and minor axis form a set of calibration coefficients which may be used to correct the measurements in the sensor frame. However, the nominal calibration method is unable to account for changes in the measurement platform's magnetic signature due to moving components or large electric currents.

In this work, moving components on the measurement platform are accounted for through a parameterized geometric field calibration method. In this method the nominal geometric calibration coefficients are parameterized by the moving components locations. Specifically, third order polynomials are estimated using gradient descent methods from a set of calibration maneuvers by minimizing the difference between the modeled and measured total field values at the calibration locations. This gradient descent optimization is further constrained through minimizing the variance between the modeled and measured vertical component of the magnetic field which enables the convergence of the method despite the limitations of available motions of the platform. The nominal coefficients are then used to form the initial conditions for a gradient descent solver which finds the parameterized equations for each calibration coefficient. The method is further extended to use as a field calibration method for magnetic compasses by including the sensor misalignment in the gradient descent solver and constraining the variance between the measured and modeled vertical magnetic field component.

The parameterized calibration method was evaluated using an autonomous underwater glider equipped with a precision low power fluxgate magnetometer. Two sets of field trials were performed using this system. The 
first set took place in December 2013 in the East Arm of Bonne Bay, Newfoundland, during which calibration runs were performed upon deployment and before recovery. For each calibration run the underwater glider performed a series of descending and ascending spirals such that the mass shifting mechanism and ballast system were each at multiple steady state locations. The second took place in the Labrador Sea in July 2014 during which two sets of calibration runs were also performed. In these trials a reduced set of runs were used with the mass shifting mechanism held at a single location to save time.

In the Bonne Bay trials the utility of the parameterized calibration method over the nominal method was shown. However, large magnetic anomalies in the nearby mountains created a challenging environment with which to interpret the accuracy of the calibrated measurements due to crustal sources being both above and below the measurement locations. For this reason a second set of trials were performed in the open ocean in deep water.

The Labrador Sea trials were performed in deep water with a reduced set of calibration spirals as a time savings measure. While the reduced set of calibration runs was deemed moderately effective the full set of calibration runs constrains the model better and is therefore recommended for future use. Additionally, the poor results from the second set of calibration runs illustrate the need for accurate measurements of the local field to calibrate against.

A parameterized geometric calibration method has been presented and demonstrated through field trials. This method is able to calibrate in the sensor frame a vector measurement of the magnetic field in the presence of moving ferrous or magnetic components whose locations can be controlled and measured. Additionally, it allows for field calibrations of marine vehicles with moving masses and limitations in their controllable degrees of freedom through a novel minimization of the variance between the measured and modeled vertical component of the magnetic field. By extending the parameterization to include the rotation bias between the vehicle and sensor frame the vehicle's heading may be computed as well.

\section{Acknowledgments}

This work was supported by the Natural Sciences and Engineering Research Council (NSERC) through the NSERC Canadian Field Robotics Network (NCFRN), the Research Development Corporation, the Marine Institute and Memorial University of Newfoundland.

\section{References}

Alonso, R. and Shuster, M. D. (2002a). Attitude-independent magnetometer-bias determination: a survey. Journal of the Astronautical sciences, 50(4):453-476.

Alonso, R. and Shuster, M. D. (2002b). Complete linear attitude-independent magnetometer calibration. Journal of the Astronautical Sciences, 50(4):477-490.

Alonso, R. and Shuster, M. D. (2002c). TWOSTEP: a fast robust algorithm for attitude-independent magnetometer-bias determination. Journal of the Astronautical Sciences, 50(4):433-452.

Bankey, V., Cuevas, A., Daniels, D., Finn, C. A., Hernandez, I., Hill, P., Kucks, R., Miles, W., Pilkington, M., Roberts, C., Roest, W., Rystrom, V., Shearer, S., Snyder, S., Sweeney, R., Velez, J., Phillips, J. D., and Ravat, D. (2002). Digital data grids for the magnetic anomaly map of North America. US Geological Survey.

Bartington Instruments (2011). Mag648 and Mag649 Low Power Three-Axis Magnetic Field Sensors, Bartington Instruments, DS2298/9, retreived July 2011.

Bronner, A., Munschy, M., Sauter, D., Carlut, J., Searle, R., and Maineult, A. (2013). Deep-tow 3C magnetic measurement: Solutions for calibration and interpretation. Geophysics, 78(3):J15-J23. 
Crassidis, J. L., Lai, K.-L., and Harman, R. R. (2005). Real-time attitude-independent three-axis magnetometer calibration. Journal of Guidance, Control, and Dynamics, 28(1):115-120.

Engels, M., Barckhausen, U., and Gee, J. S. (2008). A new towed marine vector magnetometer: methods and results from a Central Pacific cruise. Geophysical Journal International, 172(1):115-129.

Fitzgibbon, A., Pilu, M., and Fisher, R. B. (1999). Direct least square fitting of ellipses. Pattern Analysis and Machine Intelligence, IEEE Transactions on, 21(5):476-480.

Foster, C. and Elkaim, G. (2008). Extension of a two-step calibration methodology to include nonorthogonal sensor axes. Aerospace and Electronic Systems, IEEE Transactions on, 44(3):1070-1078.

Frey, J., Hawkins, J., and Thorsen, D. (2014). Magnetometer calibration in the presence of hard magnetic torquers. In Aerospace Conference, 2014 IEEE, pages 1-6, Big Sky, MT, USA. IEEE.

Furlong, M. E., Paxton, D., Stevenson, P., Pebody, M., McPhail, S. D., and Perrett, J. (2012). Autosub long range: A long range deep diving auv for ocean monitoring. In 2012 IEEE/OES Autonomous Underwater Vehicles (AUV), pages 1-7.

Gee, J. and Cande, S. (2002). A surface-towed vector magnetometer. Geophysical research letters, 29(14):151.

Honarvar, P., Nolan, L., Crisby-Whittle, L., and Morgan, K. (2013). The geoscience atlas. Report 13-1, Newfoundland and Labrador Department of Natural Resources. Geological Survey.

International Association of Geomagnetism and Aeronomy, Working Group V-MOD. Participating members, Finlay, C. C., Maus, S., Beggan, C. D., Bondar, T. N., Chambodut, A., Chernova, T. A., Chulliat, A., Golovkov, V. P., Hamilton, B., Hamoudi, M., Holme, R., Hulot, G., Kuang, W., Langlais, B., Lesur, V., Lowes, F. J., L?hr, H., Macmillan, S., Mandea, M., McLean, S., Manoj, C., Menvielle, M., Michaelis, I., Olsen, N., Rauberg, J., Rother, M., Sabaka, T. J., Tangborn, A., T?ffner-Clausen, L., Th?bault, E., Thomson, A. W. P., Wardinski, I., Wei, Z., and Zvereva, T. I. (2010). International geomagnetic reference field: the eleventh generation. Geophysical Journal International, 183(3):1216-1230.

Isezaki, N. (1986). A new shipboard three-component magnetometer. Geophysics, 51(10):1992-1998.

Kato, N. and Shigetomi, T. (2009). Underwater navigation for long-range autonomous underwater vehicles using geomagnetic and bathymetric information. Advanced Robotics, 23(7-8):787-803.

Kinsey, J. C., Eustice, R. M., and Whitcomb, L. L. (2006). A survey of underwater vehicle navigation: Recent advances and new challenges. In IFAC Conference of Manoeuvering and Control of Marine Craft, Lisbon, Portugal. Invited paper.

Korenaga, J. (1995). Comprehensive analysis of marine magnetic vector anomalies. Journal of Geophysical Research: Solid Earth (1978-2012), 100(B1):365-378.

Li, Q. and Griffiths, J. G. (2004). Least squares ellipsoid specific fitting. In Geometric Modeling and Processing, 2004. Proceedings, pages 335-340, Beijing, China. IEEE.

Markovsky, I., Kukush, A., and Van Huffel, S. (2004). Consistent least squares fitting of ellipsoids. Numerische Mathematik, 98(1):177-194.

Maus, S., Barckhausen, U., Berkenbosch, H., Bournas, N., Brozena, J., Childers, V., Dostaler, F., Fairhead, J., Finn, C., Von Frese, R., et al. (2009). EMAG2: A 2-arc min resolution earth magnetic anomaly grid compiled from satellite, airborne, and marine magnetic measurements. Geochemistry, Geophysics, Geosystems, 10(8).

Merayo, J. M., Brauer, P., Primdahl, F., Petersen, J. R., and Nielsen, O. V. (2000). Scalar calibration of vector magnetometers. Measurement science and technology, 11(2):120. 
Olsen, N., Toffner-Clausen, L., Sabaka, T. J., Brauer, P., Merayo, J. M., Jorgensen, J., Léger, J., Nielsen, O. V., Primdahl, F., and Risbo, T. (2003). Calibration of the Orsted vector magnetometer. Earth Planets and Space, 55(1):11-18.

Primdahl, F. (1979). The fluxgate magnetometer. Journal of Physics E: Scientific Instruments, 12(4):241.

Prouty, M. and Johnson, R. Small, low power, high performance magnetometers. In EGM 2010 International Workshop, Capri, Italy.

Renaudin, V., Afzal, M. H., and Lachapelle, G. (2010a). Complete triaxis magnetometer calibration in the magnetic domain. Journal of sensors, 2010.

Renaudin, V., Afzal, M. H., and Lachapelle, G. (2010b). New method for magnetometers based orientation estimation. In Position Location and Navigation Symposium (PLANS), 2010 IEEE/ION, pages 348-356. IEEE.

Rudnick, D., Davis, R., Eriksen, C., Fratantoni, D., and Perry, M. (2004). Underwater gliders for ocean research. Marine Technology Society Journal, 38:73-84.

Ryan, W. B. F., Carbotte, S. M., Coplan, J. O., O'Hara, S., Melkonian, A., Arko, R., Weissel, R. A., Ferrini, V., Goodwillie, A., Nitsche, F., Bonczkowski, J., and Zemsky, R. (2009). Global multi-resolution topography synthesis. Geochemistry, Geophysics, Geosystems, 10(3):n/a-n/a. Q03014.

Shah, V., Knappe, S., Schwindt, P. D. D., and Kitching, J. (2007). Sub-picotesla atomic magnetometry with a microfabricated vapour cell. Nature Photonics, 1:649-652.

Springmann, J. C. (2013). Satellite Attitude Determination with Low-Cost Sensors. PhD thesis, University of Michigan.

Teixeira, F. C. and Pascoal, A. M. (2008). Geophysical navigation of autonomous underwater vehicles using geomagnetic information. In 2nd IFAC Workshop Navigation, Guidance and Control of Underwater Vehicles, Killaloe, Ireland.

Tittensor, D. (2003). Creating a bathymetric and topographic matrix for the Bonne Bay region, Newfoundland, Canada. Technical report, Memorial University, Department of Physics and Physical Oceanography.

Turner, D., Anderson, I., Mason, J., and Cox, M. (1999). An algorithm for fitting an ellipsoid to data. National Physical Laboratory, UK.

Vasconcelos, J., Elkaim, G., Silvestre, C., Oliveira, P., and Cardeira, B. (2011). Geometric approach to strapdown magnetometer calibration in sensor frame. Aerospace and Electronic Systems, IEEE Transactions on, $47(2): 1293-1306$. 